Pedagogía y Saberes n. ${ }^{\circ} 54$

Universidad Pedagógica Nacional

Facultad de Educación. 2021. pp. 197-212

\title{
Una aproximación desde el uso pedagógico de ciertas polémicas públicas: proceso de paz con las FARC-EP*
}

\section{An Approach from the Pedagogical Use of Certain Public Polemics: Peace Process with the FARC-EP \\ Uma abordagem do uso pedagógico de certas polêmicas públicas: processo de paz com as FARC-EP}

Claudia Marcela Hernández Guzmán**

Hernández-Guzmán, C. (2021). Una aproximación desde el uso pedagógico de ciertas polémicas públicas: proceso de paz con las FARC-EP. Pedagogía y Saberes, (54). https//doi.org/10.17227/pys.num54-11802

* Este artículo forma parte de la tesis El dispositivo de la pedagogía de paz y sus (re)configuraciones: un estudio de su implementación por parte del Gobierno colombiano y de la guerrilla de las FARC-EP, durante el periodo 2014-2016, defendida y aprobada en junio del 2020 en el marco de la Maestría en Educación, Pedagogías Críticas y Problemáticas Socioeducativas, de la Facultad de Filosofía y Letras de la Universidad de Buenos Aires (UBA).

** Doctoranda en Ciencias Sociales, Facultad de Ciencias Sociales de la UBA; magíster en Educación. Pedagogías Críticas y Problemáticas Socioeducativas, Facultad de Filosofía y Letras, UBA. Becaria inicial en el marco del Proyecto PICT 2016-3107.

Correo electrónico: hg.claudiamarcela@gmail.com.

Código Orcid: https://orcid.org/0000-0003-0117-5323 


\title{
Resumen
}

A lo largo del último proceso de paz entre el Gobierno colombiano y las FARC-EP se vivieron diversas situaciones discursivas que cobraron carácter controversial o polémico. Estas situaciones, a la luz del plebiscito celebrado en el 2016 para refrendar el Acuerdo Final, parecieron contraproducentes para su resultado positivo. El propósito de este artículo es analizar el uso pedagógico que la Oficina del Alto Comisionado para la Paz le dio a ciertos escenarios de polémica pública los cuales tomaron una fuerza mediática significativa en el marco de la pedagogía de paz. A partir de la selección de material audiovisual y escrito, proponemos una reconstrucción de ciertos discursos contrarios al proceso de paz y de los contraargumentos elaborados por el gobierno colombiano con el fin de destacar posibles relaciones entre el "dispositivo pedagógico" y las polémicas públicas. Finalmente, señalamos que las polémicas públicas pueden ser comprendidas en clave de potencia pedagógica, que permiten la apertura de escenarios de argumentación y una consecuente resignificación de saberes y (re)configuración de identidades.

\section{Palabras clave}

pedagogía de paz; polémica pública; FARC-EP; proceso de paz; plebiscito

\begin{abstract}
Throughout the recent peace process between the Colombian Government and FARC-EP, various discursive situations acquired a controversial or polemical character. These situations, in light of the plebiscite held in 2016 to endorse the Final Agreement, seemed counterproductive to the positive outcome of the process. The purpose of this article is to analyze the pedagogical use that the Office of the High Commissioner for Peace gave to certain scenarios of public polemics, which took on a significant media force within the framework of Peace Pedagogy. Based on the selection of audiovisual and written material, we propose a reconstruction of certain discourses that were opposed to the peace process and of the counterarguments developed by the Colombian government, in order to highlight possible relations between the "pedagogic dispositive" and the public polemics. Finally, we point out that public polemics can be understood in terms of their pedagogical potential, which allow the opening of argumentation scenarios and a consequent resignification of knowledge and (re)configuration of identities.
\end{abstract}

\section{Keywords}

peace pedagogy; public polemics; FARC-EP; peace process; plebiscite

\section{Resumo}

Durante todo o processo de paz entre o governo colombiano e as FARC-EP, várias situações discursivas adquiriram um caráter controverso ou polêmico. Essas situações, à luz do plebiscito realizado em 2016 para endossar o Acordo Final, pareciam contraproducentes para o resultado positivo do processo. 0 objetivo deste artigo é analisar o uso pedagógico que o Escritório do Alto Comissariado para a Paz deu a certos cenários de polêmica pública que tomou significativa força da mídia no âmbito da Pedagogia da Paz. A partir da seleção de material audiovisual e escrito, propomos a reconstrução de certos discursos contrários ao processo de paz e dos contra-argumentos elaborados pelo governo colombiano para evidenciar possíveis relações entre o "dispositivo pedagógico" e as polêmicas públicas. Por fim, destacamos que as polêmicas públicas podem ser entendidas em termos de poder pedagógico, que permitem a abertura de cenários de argumentação e uma consequente ressignificação de conhecimento e (re)configuração de identidades.

\section{Palavras-chave}

pedagogía de paz; polêmica pública; FARC-EP; proceso de paz; plebiscito 


\section{Introducción}

Como es ya de conocimiento general, en el 2012, en La Habana, Cuba, comenzó la fase pública del proceso de paz entre el gobierno de Juan Manuel Santos (en adelante, GobjMs) y la entonces guerrilla de las Fuerzas Armadas Revolucionarias de Colombia-Ejército del Pueblo (FARC-EP). En el 2014, las delegaciones de ambas partes vieron la necesidad de responder a algunas dudas, temores y desconfianzas que estaban surgiendo sobre el proceso en ciertos sectores de la sociedad colombiana y en las filas de la guerrilla, a partir de estrategias que facilitaran la comprensión y la apropiación del proceso de diálogo. De este modo, uno de los puntos que surgió de la mesa de conversación fue implementar, lo que llamaron, la "pedagogía de paz". Esto, para generar, en primera instancia, un conocimiento sobre el proceso que se estaba llevando a cabo en La Habana, con relación a las discusiones y los acuerdos a los que se iban llegando. En segunda instancia, dicha pedagogía sería clave en acompañar la fase de implementación de los acuerdos pactados a partir de la refrendación del Acuerdo Final por parte del poder legislativo colombiano, luego del fracaso del plebiscito celebrado en octubre del 2016. En estos dos momentos se hizo pedagogía de paz en espacios y escenarios de diálogo entre la delegación del Gobierno y la población civil, por un lado, y la delegación de la guerrilla y sus filas, por el otro.

Consideramos que es posible abordar el proceso de paz desde una dimensión pedagógica-discursiva, entre otras opciones, en tanto gran parte de esta pedagogía se materializó en los discursos que circularon en los medios de comunicación masiva. En este sentido, proponemos analizar la relación entre el dispositivo pedagógico, ${ }^{1}$ especialmente en las realizaciones llevadas a cabo por el Gobierno de Colombia, y las polémicas surgidas desde el inicio de

1 Una de las hipótesis centrales de la tesis de maestría, en la cual se enmarca esta investigación, es que la pedagogía de paz, que surge como acuerdo político entre las partes negociadoras del proceso de paz de La Habana, se puede comprender como un dispositivo pedagógico, que tuvo, por lo menos, dos realizaciones: la del Gobierno colombiano y la de la guerrilla de las FARC-EP. Comprendemos el dispositivo desde los postulados foucaultianos, en la medida en la que éste implica un entramado de elementos heterogéneos, discursivos y extradiscursivos que surge para responder a una urgencia en un momento histórico específico. Así, el dispositivo no se constituye únicamente por la unión de los elementos, sino que resulta indispensable la red que entre ellos se crea. Estas relaciones, a su vez, generan vínculos de poder-saber en tanto el dispositivo está ligado a uno de los bordes del saber que nacen de él y lo condicionan. Dicha relación implica una construcción de subjetividades en la medida en que se crean, recrean o reconfiguran sujetos a partir de diferentes prácticas, discursos, saberes y ejercicios. la pedagogía de paz hasta la definición del plebiscito como mecanismo de refrendación del Acuerdo Final de Paz. Revisaremos aquellas polémicas públicas que involucraron, principalmente, al GobjMs, la guerrilla de las FARC-EP (aunque esta no haya respondido debido a las restricciones para acceder a los medios de comunicación masiva que tenía en tanto insurgencia) y a la oposición del proceso de paz, encabezada por el expresidente de Colombia y hoy senador Álvaro Uribe Vélez. $^{2}$

Hablamos de polémicas públicas ya que los diferentes contextos de reconfiguración del dispositivo pedagógico, en el marco de las realizaciones del dispositivo por parte del Gobierno colombiano y de la guerrilla, estuvieron caracterizados por posturas dicotómicas y polarizadas frente al proceso, a partir de las cuales se crearon diferentes contextos de confrontación de discursos entre los actores políticos - Gobierno, guerrilla, oposición, movimientos sociales y políticos, sociedad civil en general- - De esta manera, proponemos analizar específicamente el uso pedagógico que se le dio a las polémicas públicas, particularmente por parte de la realización del Gobierno, las cuales tomaron una fuerza mediática significativa en el marco de la pedagogía de paz. Pretendemos, entonces, indagar por el papel pedagógico de ciertas polémicas públicas, relacionadas con los cinco puntos temáticos de la agenda y el de implementación. ${ }^{3}$ Puesto entre signos de interrogación, nuestro objetivo en este artículo es responder a las siguientes preguntas: ¿la polémica motivó el ejercicio pedagógico o fue el ejercicio pedagógico el que motivó la polémica?, ¿la polémica es un elemento del dispositivo pedagógico?, ¿cómo se podrían articular en términos pedagógicos las diversas polémicas públicas que surgieron del proceso de paz con las realizaciones del dispositivo pedagógico?

2 Como se mencionó más arriba, nos centraremos en las polémicas públicas surgidas entre el Gobierno nacional, las FARC-EP y la oposición encabezada por el uribismo en la medida en que el corpus de análisis lo tomamos de los registros virtuales de los medios de comunicación nacionales y de la mesa de negociación (Oficina del Alto Comisionado para la Paz, particularmente). A pesar de que reconocemos que hubo hay-muchos otros actores políticos en oposición al entonces Gobierno nacional, estas voces no contaron con tanta visibilidad por parte de estas plataformas virtuales. Adicionalmente, como veremos a lo largo del artículo, el GobjMs decidió responder, casi que de manera exclusiva y a lo largo del periodo seleccionado, a los postulados del uribismo.

3 Recordemos que la agenda de negociación estuvo compuesta por los siguientes puntos temáticos: desarrollo agrario integral, participación política, fin del conflicto, narcotráfico y consumo de drogas ilícitas, víctimas e implementación. 
Presentaremos los contenidos en cinco partes. En la primera, propondremos una breve reconstrucción de la comprensión de la pedagogía de paz, en tanto dispositivo, por parte del GobjMS y de las FARC-EP, respectivamente. En la segunda parte, presentaremos el marco teórico a partir del cual abordamos las polémicas públicas y su uso pedagógico. En la tercera, daremos cuenta del contexto de polémica pública que hubo durante todo el proceso de paz hasta el plebiscito para la refrendación del primer Acuerdo Final, en el marco del dispositivo de pedagogía de paz. En la cuarta, analizaremos unas polémicas particulares, las cuales influyeron en ciertos discursos retomados por la pedagogía de paz en sus materiales escritos y audiovisuales. Y, en la quinta parte, concluiremos con una propuesta de análisis sobre los modos en que el uso de las polémicas presentadas potenció el carácter pedagógico del dispositivo.

\section{Breve reconstrucción de la comprensión de la pedagogía de paz por parte de las partes negociadoras}

Desde el inicio, el dispositivo se configuró para dar cuenta, por un lado, del proceso histórico por el que estaba pasando Colombia durante esos años (y que hoy sigue) y, por el otro, para responder a la urgencia de fortalecer el proceso frente a las posiciones contrarias a la manera como se estaba proponiendo construir la paz desde la mesa de negociación. El dispositivo se fue reconfigurando desde la comprensión particular de la pedagogía de paz que cada uno de estos actores redefinió a lo largo del periodo seleccionado. La definición de pedagogía de paz que quedó consignada en el Acuerdo Final de Paz es:

Sistema Conjunto de Comunicación y Pedagogía del Acuerdo Final y divulgación sobre los avances de la implementación [el cual] incluye piezas pedagógicas para comunicar los contenidos del Acuerdo Final [...] y para difusión y pedagogía del inicio de la implementación del Acuerdo Final. (Gobierno nacional y FARC-EP, 2016, p. 191)

Adicionalmente, la guerrilla complementó la definición de la pedagogía como una oportunidad de enseñanza para que la guerrillerada entendiera el proceso de negociación, pero también como una posibilidad para fomentar la cultura, el deporte y la integración de su militancia guerrillera.

A pesar de los diferentes matices entre el Gobierno y la guerrilla, estos dos actores coincidieron en que la pedagogía de paz debía facilitar la comprensión de una concepción compartida del proceso de las negociaciones y contribuir a generar condiciones que permitieran implementar los acuerdos a los que se llegaran en La Habana. Otra semejanza remite a las "funciones estratégicas" del dispositivo para garantizar un nivel suficiente de legitimidad del proceso de paz, por medio de la apropiación de los acuerdos para lograr su refrendación por medio del voto popular. En este orden de ideas, la definición del objetivo de la pedagogía de paz también representó una semejanza. Estos dos actores coincidieron en que era necesario aclararle a la sociedad civil y a la militancia guerrillera que en La Habana se estaba negociando el fin del conflicto, pero que la paz se construye con toda la población.

En lo que respecta a la realización del dispositivo llevada a cabo por el Gobierno, observamos que este optó por desplegar una numerosa serie de elementos de distinta índole y tono para lograr dirigirse a un destinatario igualmente amplio y heterogéneo: la sociedad civil urbana y rural. Para ello, reprodujo los acuerdos de paz parciales, a medida que se iban consolidando a través de documentos "originales", documentos de prensa, cartillas, infografías, videos didácticos, videos explicativos y encuentros de convocatoria abierta y cerrada. Las variaciones en los formatos de los materiales de difusión y espacios de participación también se vieron reflejadas en los contenidos. A medida que fue avanzando el proceso de negociación y los acuerdos, la realización del dispositivo fue tomando más consistencia y el carácter pedagógico también se fortaleció. Esto se puso en evidencia, por ejemplo, en las cartillas que cada vez tuvieron menos texto y más imagen o en la producción de nuevos videos cortos y concisos. En algunos discursos decidieron ceñirse a los acuerdos y en otros optaron por responder a correlatos que estaban surgiendo en la esfera pública. Resulta interesante, entonces, reparar en las redes o los vínculos que se tejieron entre los diferentes elementos constitutivos del dispositivo: los materiales de difusión, los discursos, los escenarios, las convocatorias, los y las destinatarias, los y las enunciadoras. Así, lograron establecer una relación permanente y dinámica entre el relato que se estaba construyendo en La Habana y el que se construía como correlato en Colombia con el fin de legitimar un proceso que buscó el fin del conflicto y, por lo tanto, un país en paz.

En la realización del dispositivo de pedagogía de paz de las FARC-EP, el hecho de que el destinatario, en principio, fuera otro (la guerrillerada), permitió otros vínculos entre sus elementos. A este respecto, observamos que los escenarios pedagógicos que este actor dinamizó estuvieron acompañados por 
actores externos a la guerrilla (países garantes del proceso, la onU y el Gobierno colombiano), lo cual, probablemente, coartó sus discursos. No obstante, notamos una búsqueda por llevarles tranquilidad a sus propias filas con relación a su futuro en la vida civil, manteniéndose vigente al mismo tiempo su concepción de "la nueva Colombia", ahora desde la legalidad. Lo anterior, teniendo en cuenta que la difusión de los espacios de participación y de los materiales realizados por las FARC-EP estuvo siempre limitada, en tanto actor armado al margen de la ley, durante todo el proceso de paz en general y, en particular, en lo referido al dispositivo pedagógico. De esta manera, se evidencia un dispositivo pedagógico constituido por un conjunto diverso de materiales, escenarios y de discursos tanto como de destinatarios y objetivos de intervención.

\section{Hacia una comprensión (aproximación) de las polémicas públicas y sus usos pedagógicos}

De la mano con Amstutz et ál. (2005), quienes proponen comprender el conflicto como una situación estimulante para la formación del ser humano, y teniendo en cuenta adicionalmente los postulados de Amossy $(2016,2017)$ sobre la polémica como un ejercicio argumentativo que permite construir nuevas posibilidades, a lo largo de este texto procuramos visibilizar cómo un contexto de polémica pública permanente, que podría parecer adverso al proceso de paz y, específicamente, a un ejercicio pedagógico que se venía realizando por parte, por lo menos, de los dos actores protagonistas de este, permitió, por el contrario, alimentar y dinamizar el dispositivo pedagógico de paz.

Como lo señalamos antes, hemos decidido abordar el análisis de las polémicas públicas en concordancia con la comprensión positiva del conflicto que se propone desde algunas visiones de la pedagogía de paz (Amstutz et ál, 2005), ya que invitan a realizar una lectura "positiva" del disenso y del conflicto en términos de posibilidad de argumentación; es decir, como un mecanismo que permite el pensamiento, la creación o el refuerzo de identidades y la (re)construcción de saberes que se puedan llevar a cabo en aras de una pedagogía de paz. De esta forma, para el análisis de las polémicas públicas enmarcadas en el dispositivo pedagógico en cuestión, retomamos los postulados de Amossy $(2016,2017)$ sobre la teoría del dissensus, quien pone en evidencia que los disensos, en el contexto de las discusiones públicas democráticas, son más comunes de lo que se ha querido mostrar. Consideramos oportuno el énfasis que la autora realiza del marco democrático en el que, por un lado, se ha caracterizado la teoría de la argumentación y la retórica y, por el otro, en el que ella desarrolla sus postulados sobre la polémica. Este aspecto es relevante para el caso que se estudia, en la medida en que Colombia se ha caracterizado por vivir en democracia a pesar del conflicto armado interno, lo cual ha sido utilizado como argumento para la salida pacífica, negociada y consensuada de los conflictos en general y, en particular, del armado, a pesar de las múltiples y fuertes estrategias de contraofensiva militar que también se han llevado a cabo de manera simultánea.

Amossy (2017), en Apología a la polémica, propone comprender la polémica pública como un ejercicio racional y argumentativo, a partir del cual se pone en cuestión la idea de que esta únicamente recurre a la violencia verbal y los sentimientos para deslegitimar otras posturas (políticas). En este sentido, la autora sostiene que la polémica pública cumple un rol constitutivo en el régimen democrático, en clave de lo que ella llama la retórica del dissensus, "cuyo objetivo último no es la búsqueda del acuerdo, cuyo modelo no es el diálogo, y [...] cumple no obstante funciones importantes en el espacio público" (2016, p. 25). En contraposición a las teorías clásicas de la argumentación y la retórica, se refiere a la manera no excepcional de gestionar los conflictos en los espacios democráticos pluralistas por medio de la argumentación, los cuales denomina dissensus y agon. Subraya, así, que la polémica implica confrontación de argumentos, puesto que busca defender un punto de vista divergente. Con respecto al carácter público de la polémica, Montero (2016), en sintonía con Amossy, aclara que la cuestión que genera el conflicto debe ser de interés general, al alcance de la ciudadanía, "común y compartida por una comunidad más o menos vasta de individuos" (2016, p. 15).

Amossy plantea que, a partir de la polémica pública, se busca gestionar un conflicto en un marco de disenso. Dicha polémica se crea cuando existen, por lo menos, dos respuestas distintas a una misma cuestión, lo que implica un ejercicio de justificación de suposiciones a partir de fundamentos. Esta exposición de argumentos conduce a grados variados de confrontación verbal. Así, la polémica se caracteriza porque es un debate que confronta puntos de vista opuestos sobre una cuestión de interés público y se lleva a cabo mediante tres procedimientos que están estrechamente ligados entre sí: la dicotomización, la polarización y el descrédito hacia la(s) otra(s) persona(s) y, finalmente, puede estar acompañado de pasión y violencia verbal. Esto es, no hay una pretensión por lograr un acuerdo sobre el conflicto en cuestión; no obstante, su gestión puede darle cabida a la posibilidad de razonar. Es una 
disputa discursiva, a partir de argumentos, ya que es necesario un proceso de pensamiento para ganar el conflicto verbal. En términos de Montero, el interdiscurso que constituye la polémica "puede pensarse [...] como un campo de discursos en tensión que rodean y determinan una determinada formulación, enunciado o discurso: de allí que la polémica sea siempre una posibilidad en potencia" (2016, p. 11). En definitiva, la polémica invita a más voces a unirse a la discusión, a participar con argumentos.

En este orden de ideas, y en la medida en que los contextos de reconfiguración del dispositivo se caracterizaron por posturas dicotómicas y polarizadas frente al proceso, y generaron ciertas polémicas públicas relacionadas con los puntos de la agenda de negociación, afirmamos que dichas polémicas públicas tuvieron un papel pedagógico relevante. Decimos entonces que hubo un uso pedagógico de los disensos, de las polémicas públicas, en tanto la realización del dispositivo por parte del GobjMs retomó los argumentos de la oposición y procuró, a partir de argumentos, defender los acuerdos de La Habana y reforzar su legitimidad frente al pueblo colombiano.

Lo anterior, de la mano con la concepción del dispositivo desde el campo pedagógico (Grinberg, 2008, 2011; Langer, 2013; Palumbo, 2014, 2017; Simons y Masschelein, 2017), la cual propone comprender los dispositivos pedagógicos como una articulación de prácticas discursivas y no discursivas, de saberes, de procesos y de estrategias que convergen en los espacios-momentos de formación, en el encuentro entre, por los menos, dos sujetos, donde se crean subjetividades y se despliegan relaciones de saber-poder.

El despliegue de estas relaciones de saber-poder se dan en la medida en que el dispositivo está ligado a uno de los bordes del saber que nacen de él y lo condicionan, lo cual implica una construcción de subjetividades puesto que se crean, recrean o reconfiguran sujetos a partir de diferentes prácticas, discursos, saberes y ejercicios. En este sentido, planteamos una relación indisociable entre política y pedagogía ya que se genera un encuentro de subjetividades, y de construcción de subjetividades, en torno a la socialización de valores, saberes y proyectos que reproducen o transforman estados de cosas (Palumbo, 2017).

\section{Contexto general de las polémicas durante el proceso de paz}

Desde que se presentó públicamente la mesa de conversaciones para la paz en el 2012, las tensiones no tardaron en salir a la luz. Para ese momento, el Gobjus había cumplido dos años de su primer mandato y las distancias políticas del presidente con su antecesor, Álvaro Uribe Vélez, cada vez eran más profundas. Como es sabido, Uribe Vélez se convirtió, por lo menos desde su primer mandato como presidente de Colombia (2002-2006), en una figura política con una importante incidencia en la opinión pública nacional y regional. Si bien Santos compartía - y comparte- muchos de los principios políticos liderados por Uribe, esta relación se fue debilitando progresivamente una vez Santos asumió la presidencia. Dicha relación fracturada se pudo percibir en las polémicas públicas permanentes entre estos dos actores, animadas por las críticas a las decisiones que tomaba Santos en su administración, las cuales variaban en sus grados de polarización según el tema en cuestión. Juan Manuel Santos, y por extensión, el proceso de paz en el que se inscribe la realización del dispositivo pedagógico por parte del Gobierno, tenía más contrincantes a pesar de las alianzas electorales pasadas: los demás partidos políticos, algunos sectores de la Iglesia católica y evangélica, los movimientos sociales y políticos de izquierda y, claro, la guerrilla de las FARC-EP. No obstante, las polémicas desatadas con estos últimos actores mencionados fueron menos y de menor intensidad ya que, entre otros motivos, contaron con poca o ninguna cobertura mediática.

Asimismo, las tensiones entre el gobierno y la guerrilla estuvieron presentes durante toda la fase exploratoria, como es de esperarse, y se mantuvieron durante todo el proceso de paz. Sin embargo, estas tensiones se fueron matizando o profundizando en función de los temas que se iban negociando en La Habana y la coyuntura política del país. Una de las primeras controversias, que desembocó en polémica pública, fue a propósito de la agenda acordada y con la cual dieron a conocer la fase pública de la mesa de conversación. Después de que Juan Manuel Santos anunciara el inicio de la fase pública del proceso de paz y explicara el funcionamiento de la mesa de conversaciones, a través de una alocución presidencial ${ }^{4}$ transmitida por el canal nacional institucional y después reproducida por los demás canales de televisión nacionales, la guerrilla publicó un video ${ }^{5}$ — dirigido especialmente a la guerrillerada y a sus bases- en el que su comandante, alias Timochenko, también anunció el inicio de las rondas de negociación y su compromiso con la paz.

\footnotetext{
4 Véase la alocución presidencial completa en https://www. youtube.com/watch?v=6GKFKen0 $\mathrm{mi} 8$

5 Véase el video completo en https://www.youtube.com/ watch?v=RjvA5I8r6TU
} 
No obstante, dado que la dirección de las FARC-EP consideró que la delegación guerrillera había cedido mucho ante el Gobierno, con lo cual no estaba completamente satisfecha con la agenda de negociación acordada, Timochenko, en este video que después reprodujeron parcialmente los canales nacionales, fue más allá de lo establecido en la mesa de conversaciones y se refirió a otros temas que ocasionaron la polémica pública. El jefe guerrillero recordó el proceso de paz fallido con el expresidente Andrés Pastrana (1998-2002); mencionó algunos temas a los que no pudieron llegar a acuerdo con el gobierno actual, como el cese al fuego y de hostilidades bilateral desde el inicio; habló sobre el rol de las fuerzas armadas de Colombia y, finalmente, hizo referencia al papel de Cuba y Venezuela en el proceso de acercamiento entre estos dos actores. Estos cuatro temas, en particular, ocasionaron controversias en la esfera pública, además de que Timochenko apareció con el uniforme guerrillero y utilizó un lenguaje que se considera en Colombia como de extrema izquierda.

Así, algunas de las polémicas surgieron, por un lado, porque la oposición de derecha al proceso de paz empezó a alegar que la paz se estaba construyendo a espaldas del país, pues en las dos comunicaciones se hizo énfasis en el tiempo exploratorio presentado como exitoso. Además, comenzó a circular la idea de que iba a haber una agenda paralela y clandestina, lo cual podría implicar "entregarle el país a las FARC". La alusión, por parte de la guerrilla, al rol de las fuerzas militares colombianas, al resaltar positivamente su trabajo, también generó incomodidades en los sectores de derecha y ultraderecha. Finalmente, el reconocimiento a la gestión de Venezuela, en particular, fue uno de los temas más sensibles. Probablemente, la primera acción política pública que llevó a estas fracturas políticas entre Santos y su antecesor fue la recomposición de las relaciones diplomáticas con Venezuela ${ }^{6}$ en el 2010, hecho que fue utilizado por Uribe, y la oposición de derecha en general, para asegurar que Colombia se iba a convertir en un Estado "castrochavista".

Por otro lado, la citada intervención de las FARC-EP mediante la difusión del video de Timochenko también generó tensiones con el gobierno, las cuales se prolongaron unas semanas más, con la filtración, por parte de Telesur y RCN Radio, del primer borrador conjunto con el que anunciarían oficialmente la instalación de la mesa de conversaciones. Estos hechos

6 A finales de la segunda administración de Álvaro Uribe Vélez, el 22 de julio del 2010, el Gobierno de Venezuela rompió relaciones con Colombia, luego de que el expresidente Uribe presentara ante el Consejo Permanente de la oEA una denuncia por supuesta presencia de campamentos guerrilleros en territorio venezolano. profundizaron las desconfianzas que había entre los dos actores del proceso de paz, alimentadas por las opiniones públicas desfavorables. Empero, y en aras de que no se quebrara el proceso que iba a iniciar, hubo esfuerzos de las dos partes para que los desacuerdos entre ellos no se convirtieran en polémicas públicas o para que dichas polémicas desaparecieran pronto, llegando a consensos sobre los temas que se consideraban delicados. Luego, en agosto del 2013, las FARC-EP anunciaron un receso del proceso de paz en vista de que el presidente de la República había presentado un proyecto para refrendar los acuerdos en el marco de las elecciones presidenciales del 2014. Esta decisión no la compartió la guerrilla porque, en su opinión, el proceso de negociación no podía convertirse en una bandera electoral, con lo cual se pondría en riesgo el proceso de paz, ya que el uribismo tenía cada vez más fuerza por su rechazo a este. Por su parte, Santos pidió a su delegación de La Habana regresar al país.

Hemos incluido en este breve contexto algunas situaciones que anteceden el recorte temporal seleccionado para el análisis acá propuesto, en la medida en que las consideramos pertinentes, puesto que ponen en evidencias aquellos desacuerdos entre las partes negociadoras. Adicionalmente, se puede introducir un hito del proceso de negociación en general, y de la pedagogía de paz en particular, relacionado con la polémica pública, el cual corresponde a las elecciones presidenciales del 2014. Sobre la relación de la polémica pública con las coyunturas electorales, Catherine Kerbat-Orecchioni sostiene que el objetivo de un debate es el de predominar sobre el/la contrincante, por lo que:

[...] los debates mediáticos sobre temas políticos, y más específicamente, los debates electorales, en los que la polémica es llevada a su grado extremo en la medida en que el propósito último del intercambio, que determina la totalidad del comportamiento de los locutores, es el de lograr vencer al interlocutor en la interacción, con el objetivo de hacerlo también en las urnas. (2016, p. 98)

Si bien en el marco de dichas elecciones presidenciales se generaron alianzas del Gobierno de turno con diferentes partidos políticos, también hubo tensiones entre el Gobierno y el resto de los partidos, motivadas por el hecho de que no compartían completamente la manera de concebir la política del entonces presidente Santos, ni confiaban en su figura, así como tampoco en la guerrilla de las FARC-EP. No obstante, estas discusiones, debates y, en algunos casos, polémicas públicas, no tuvieron la fuerza ni la intensidad de las que protagonizó la oposición de derecha. 


\section{Polémicas públicas y sus usos pedagógicos en la realización del dispositivo por parte del Gobierno colombiano}

En la cartilla Entérese del proceso de paz, publicada por la Oficina del Alto Comisionado para la Paz (OACP), desde el equipo negociador del Gobierno, con el aval de la guerrilla, armaron una suerte de mapa sobre el proceso de paz. La primera versión ${ }^{7}$ está dividida en ocho partes, cuyo séptimo apartado es un comunicado firmado por el jefe de la delegación del gobierno, Humberto de la Calle, sobre los mitos y las realidades del proceso de paz. De la Calle justifica este comunicado diciendo al principio que "Algunos opositores al proceso de diálogo en La Habana han puesto a circular versiones falsas y mitos inverosímiles sobre su verdadero alcance. Vamos a esclarecer enseguida las principales leyendas" (OACP, 2014, p. 13). Enumera, entonces, cinco "versiones" sobre los alcances del proceso de paz y, renglón seguido, las aclara. Estas versiones son: en La Habana se le está entregando el país a las FARC-EP; va a haber una reducción de las fuerzas militares y recorte de su estatus luego de la terminación del conflicto; la reforma rural integral afectará la propiedad privada; las conversaciones son clandestinas, a espaldas del país, y habrá impunidad para los responsables de crímenes de lesa humanidad.

Destacamos, así, el uso y la resignificación de algunos discursos desfavorables al proceso de paz que estaban circulando en la esfera pública, los cuales fueron retomados en el documento titulado "Mitos y realidades sobre el proceso de conversaciones", publicado en junio del 2014 en las cartillas Entérese del proceso de paz y en Todo lo que debería saber del proceso de paz. Visión, realidades y avances en las conversaciones que adelanta el Gobierno nacional en La Habana. Después de dos años, entre mayo y junio del 2016 (previo al plebiscito), la OACP publicó once videos cortos llamados Mitos urbanos sobre el proceso de paz en su página de YouTube, a partir de los cuales pretendía "desmentir" dichos "mitos urbanos", que remitían a algunos de los temas polémicos a los que se refieren en las cartillas citadas. Para el estudio acá propuesto, decidimos tomar estos dos materiales difundidos por el Gobierno de Colombia, ya que

7 De esta cartilla, la OACP elaboró por lo menos tres ediciones. Desde el cambio de gobierno en el 2018, la página web de la OACP fue modificada y gran parte de la información relacionada con la pedagogía de paz del reciente proceso de paz con las FARC-EP fue eliminada o distribuida en otras páginas web institucionales, con lo cual acceder a este material hoy es más complicado. permiten dar cuenta del ambiente polémico que se mantuvo durante todo el proceso de paz. A partir de estos documentos, a continuación, propondremos una reconstrucción de ciertas polémicas públicas que giraron alrededor de estos "mitos", siguiendo la denominación elegida por el dispositivo pedagógico en la realización del Gobierno. Para dar cuenta de los contradiscursos, tomamos algunos registros de la prensa colombiana como parte del corpus de documentos en análisis.

\section{"Le van a entregar el país a las FARC" y "El proceso de paz llevará a un estado castrochavista"}

La primera polémica a la que hacemos referencia es aquella que la OACP presenta como el mito respecto a que en La Habana "le van a entregar el país a las FARC" y "el proceso de paz llevará a un Estado castrochavista". Estos enunciados fueron repetidos en diferentes ocasiones por la oposición de extrema derecha desde que se dio a conocer el proceso de negociación con la guerrilla y después cobraron más peso cuando se divulgó el tratamiento que recibiría el acuerdo sobre la Jurisdicción Especial para la Paz. Desde el inicio del proceso de negociación, se afirmó en numerosas ocasiones que Juan Manuel Santos le estaba entregando el país a la guerrilla o al castrochavismo. Esta aseveración fue motivada por diferentes situaciones; por ejemplo, en el marco de las elecciones presidenciales del 2014, estas afirmaciones se intensificaron.

A partir de dichas elecciones presidenciales, las polémicas públicas que perduraron en el tiempo, fueron protagonizadas, especialmente, por los miembros del partido de oposición al Gobierno, el Centro Democrático, y la administración de Santos. Retomando los enunciados arriba citados, en uno de los eventos públicos electorales de este último candidato, en una intervención del expresidente, este aseguró que la propuesta de Oscar Iván Zuluaga era "todo lo contrario del castrochavismo de Maduro, Santos, Petro y las Farc" ("Uribe", 2014). No era la primera vez que Uribe se refería al castrochavismo, ni tampoco la última, puesto que logró hacer de este concepto una forma de crear adhesiones y simpatías con una parte de la población colombiana. Teniendo en cuenta el consenso social colombiano que rechaza la idea del castrismo en Cuba y del chavismo en Venezuela, ubicar a Maduro, Santos, Petro y las FARC-EP en el mismo grupo de castrochavistas no fue indiscriminado. Es necesario recordar que Uribe ha sido uno de los mayores opositores de los Gobiernos de Cuba y después también de los venezolanos liderados por 
Chávez y luego por Maduro; oposición que se convirtió en política exterior de bloqueo, de la cual también participó, en su momento Juan Manuel Santos como ministro de Hacienda y de Defensa. No obstante, en vista de que una de las primeras acciones presidenciales del Gobjms fue recomponer las relaciones diplomáticas con este vecino país, la grieta con su antecesor se empezó a profundizar. Dadas estas distancias políticas, Uribe comenzó a señalar a Santos como castrochavista con la intención, entonces, de poner su reputación política en cuestión.

Sobre estas aseveraciones, Frank Pearl, plenipotenciario del Gobierno en la Habana, en el video ¿El proceso de paz llevará a un Estado castrochavista? publicado el 12 mayo 2016, afirmó que:

Desde el primer día que nos sentamos con las Farc, quedó claro que acá no están en juego ni el modelo político ni el modelo económico. Los acuerdos que hemos logrado en el tema rural y en el tema político están dentro de nuestra constitución y de nuestro sistema económico. Aquí lo que hay son oportunidades de prosperidad, crecimiento e inversión con seguridad. (OACP, 2016)

Argumenta, entonces, que el Gobierno limitó los temas que se iban a discutir en La Habana, que los acuerdos a los que se han llegado están dentro de la marco legal y constitucional de Colombia, que no se rige con los principios de Cuba ni de Venezuela, y que, de hecho, lo que se pretende es fomentar el crecimiento económico del país.

Un año más tarde, en el 2015, en La Habana llegaron al acuerdo de crear el mecanismo de Justicia Transicional, en el marco de la Jurisdicción Especial para la Paz, el cual incluyó un tribunal para juzgar a los actores del conflicto armado que participaron en crímenes de lesa humanidad, incluidos la guerrilla, la fuerza pública nacional, los paramilitares y demás actores vinculados al conflicto. En este contexto, el fiscal general de la nación de ese entonces, en una comunicación pública sobre este mecanismo, aseguró que el expresidente y senador Uribe "podría ser investigado por el Tribunal para la Paz por hechos registrados cuando fue gobernador de Antioquia" ("Le van a entregar el país", 2015), ${ }^{8}$ lo cual generó, como era de esperarse, reacciones inmediatas. La primera respuesta de Uribe fue: “[l]e van a entregar el país a las Farc, van a hacer este daño por meterme a mí a la

8 El Tribunal Superior de Medellín, Antioquia, inició una investigación en contra de Álvaro Uribe por presunta responsabilidad en el caso de las masacres realizadas por paramilitares en las localidades de La Granja (1996) y de El Aro (1997), las cuales están ubicadas dentro del departamento que gobernaba Uribe, entre 1995 y 1997. cárcel, valiente gracia, esa es la paz imperfecta que están buscando" ("Le van a entregar el país", 2015). En el marco de este acuerdo, el expresidente retomó la anterior polémica, a partir de la cual la oposición de derecha denunciaba que el proceso de paz solo iba a servir para entregarle el país a la guerrilla y a la ideología castrochavista. En este caso, la oposición argumentó, por un lado, que el mecanismo se creó para juzgar a una sola persona y que este hecho demostraba la imperfección del proceso, resignificándolo con la fórmula "paz imperfecta".

Mediante material pedagógico, el Gobierno reforzó los argumentos a favor del proceso de paz para refutar las ideas que estaban utilizando y buscando instalar desde la oposición. Sobre entregarle el país a la guerrilla a través del proceso de paz, afirmó:

Nada más alejado de la realidad. En primer término, las conversaciones se han venido ciñendo estrictamente a los puntos pactados en el Acuerdo General de La Habana [...] estos puntos son eficientes y suficientes para poner fin al conflicto y abrir, sin armas, una etapa amplia de transformaciones con garantías plenas para el movimiento que surja de las FARC y entre en la política.

En segundo lugar [...] no hay que confundir las intervenciones habituales de las FARC a través de las cuales dan a conocer las más disímiles posiciones políticas, con lo que ocurre en la Mesa de Conversaciones.

En tercer lugar, lo pactado hasta ahora en los dos primeros puntos de la Agenda, si bien contiene puntos trascendentales para una reforma rural integral y para una nueva apertura democrática, está inscrito fielmente en el ideario del Estado Social de Derecho (OACP, 2014).

El primer argumento hace referencia a los términos que se definieron en el marco del Acuerdo General de La Habana del 2012, los cuales se dieron a conocer desde el principio. El siguiente argumento está dirigido a recordar que el Gobierno sigue siendo opuesto a las FARC-EP, a pesar del proceso de paz. Eligen un argumento que le resta importancia al interlocutor de las negociaciones dado que, con esta afirmación, el Gobierno se mueve del lugar de "iguales" con la guerrilla y busca generar simpatía con la oposición, a quien va dirigido el discurso. Y terminan la argumentación aclarando que los dos acuerdos a los que han llegado hasta la fecha (junio del 2014) se inscriben "fielmente en el ideario del Estado Social de Derecho", lo cual correspondería a un contrargumento sobre la idea de un Estado castrochavista. 
En el video titulado ¿En La Habana los negociadores del Gobierno le están entregando el país a las Farc?, publicado en junio del 2016, el general retirado Jorge Enrique Mora, quien era plenipotenciario del Gobierno en La Habana, inicia la intervención con un argumento de autoridad ("42 años de mi vida militar se los dediqué a mi ejército"), y resalta sus años en las Fuerzas Armadas de Colombia y el papel de los militares en la defensa del territorio nacional. Continúa diciendo que, gracias al trabajo de los militares, hoy las FARC-EP están sentadas en La Habana. Con este segundo argumento, desplaza el énfasis hacia el ejercicio de las fuerzas militares y deja a un lado el aspecto político. Luego, subraya que el fin del conflicto se logrará con "la generosidad de los colombianos [la cual] permit[irá] que las Farc se reintegren a la sociedad". Agrega que "las armas de las FARC desaparecerán para siempre, para que nunca jamás sean utilizadas", que "las FARC se reincorporan para respetar a la sociedad, a las instituciones y a nuestro sistema democrático" y termina su intervención acudiendo a la esperanza, cuando afirma que "estamos en La Habana para una Colombia con futuro, una Colombia sin conflicto".

Vemos, entonces, el uso pedagógico que se le dio a estas primeras polémicas públicas. Aun cuando los argumentos que utiliza el Gobierno están respaldados por el mismo Acuerdo (el cual se está criticando desde los argumentos que polemizan con este), procura explicarlos utilizando otro lenguaje, otras palabras, y a partir de diversos formatos que le permitan reafirmar el mensaje a los diferentes sectores de la sociedad. Por ejemplo, el de la cartilla implica un lenguaje sencillo y explicativo mientras el del video busca garantizar una proximidad e inmediatez en el mensaje.

Lo que observamos a partir del uso pedagógico de estas polémicas públicas particulares es un distanciamiento por parte del Gobierno de la postura política con la guerrilla, creando un "nosotros" - el Gobierno-que se sienta en una mesa de negociación con un "ellos" - la guerrilla-. Esto es, se reafirma una identidad, que busca legitimidad por parte de la población colombiana en general y con la oposición al proceso de paz en particular. Esta aceptación se busca, además, por medio de la afirmación de uno de los principios que rigen el proceso de paz, relacionado con el respeto por la propiedad privada y la inversión. En consecuencia, polémica, dispositivo y pedagogía se entrecruzan en la disputa por la construcción de identidades individuales y colectivas (de un "nosotros").

\section{"Impunidad total para las FARC-EP"}

En mayo del 2016, desde La Habana, las delegaciones dieron a conocer el mecanismo para garantizar la continuidad y aplicación del Acuerdo de Paz ${ }^{9}$ mediante un comunicado conjunto en el que anunciaron que

[h]emos llegado a un acuerdo para brindar seguridad y estabilidad jurídica al acuerdo final, para asegurar su introducción al ordenamiento jurídico colombiano y para dar garantías de cumplimento de dicho acuerdo final, tanto conforme al derecho interno colombiano como conforme al derecho internacional. (Gobierno de Colombia y FARC-EP, 2016)

Este acuerdo ocasionó una de las polémicas públicas más fuertes del proceso de paz. Además de la trascendencia del asunto, este incluyó acciones que se debían llevar a cabo inmediatamente para que fuera un hecho el carácter constitucional, como presentar la ley correspondiente ante el poder legislativo del país. En este caso, como en los anteriores, las reacciones que más resonaron fueron, de nuevo, las de Uribe, quien se encontraba en Miami (EE. UU.) en un evento sobre la democracia en las Américas. En una entrevista que le hizo el noticiero colombiano NTN24 sobre dicho acuerdo, él respondió:

\begin{abstract}
¿Cómo podemos aceptar que el gobierno colombiano lo ponga [a las FARC-EP] en pie de igualdad con nuestro estado democrático? ¿Cómo podemos aceptar que el gobierno colombiano y ese grupo terrorista se auto-proclamen constituyentes, cambien nuestra constitución para darle impunidad a ese grupo terrorista? Eso tiene todos los riesgos jurídicos porque, así involucren al Consejo de Seguridad [de la oNU], en cualquier momento esa impunidad total que se le ha dado a este grupo puede activar la jurisdicción de la Corte Penal [Internacional] y, desde el punto de vista interno, ese es un acuerdo que en algún momento se puede derogar, como derogaron las leyes de punto final de Chile, Argentina o, en (sic), aplican la del Salvador. (NTN24, 2016, 13 de mayo)
\end{abstract}

En esta intervención, el expresidente recurre a algunos temas que habían ocasionado controversias durante el proceso de paz, como la posibilidad de llamar a una constituyente para refrendar el Acuerdo

9 Decidieron elevar los acuerdos a la categoría de acuerdo especial, figura contemplada en el derecho internacional humanitario, cuyo objetivo radica en darle vía libre a las partes para terminar o humanizar la guerra. Adicionalmente, para incorporar el Acuerdo al ordenamiento jurídico colombiano, se propuso hacerlo a través del bloque de constitucionalidad, el cual implica que dicho Acuerdo entraría a hacer parte de la Constitución Política de Colombia por vía de uno de sus artículos (el 93), para así, crear un artículo transitorio. 
Final, iniciativa que había sido impulsada por el Centro Democrático ${ }^{10}$ y por las FARC-EP, entre otros, al inicio de las rondas de negociación. No obstante, en esta entrevista, Uribe invierte esa idea, y la presenta como negativa. Sin dejar de lado, entre otras cosas, que lo que estaban proponiendo desde La Habana no era una asamblea constituyente sino una ley con aval constitucional. En esta línea, el expresidente y senador maximiza a tal punto la decisión de volver constitucional el Acuerdo Final, que, para tornar absurdo el Acuerdo, lo asimila con una constituyente. En esta vía de ridiculizar el proceso de paz, asegura que el Gobierno y los "terroristas" se autoproclamaron constituyentes, lo cual agrava la situación pues implica que pasaron por encima del pueblo colombiano. Por otro lado, se refiere a la guerrilla como "terroristas". Con esta caracterización de las FARC-EP, les quita el estatus de sujeto político con el que se puede negociar. En consecuencia, con respecto al argumento sobre el peligro que puede representar ese mecanismo de seguridad jurídica al permitir, eventualmente, el ingreso de la Corte Penal Internacional a Colombia pretende mostrar lo débil de este acuerdo y su futuro fracaso. En una entrevista posterior, en el mismo noticiero, Uribe especifica este punto:

La ley de impunidad total de 1993 de El Salvador se cayó hace pocas semanas. 23 años después. Aquí puede ocurrir lo mismo, porque Colombia es miembro de la Corte Penal Internacional, que exige reclusión para esos delitos. Colombia es miembro del Tratado Interamericano de Derechos Humanos, que exige sanciones severas para esos delitos. (NTN24, 2016, 5 de agosto)

La mención a la derogación de las leyes de punto final en los tres países señalados, Chile, Argentina y El Salvador, pretende alertar a la población colombiana del fracaso inminente que representa la medida adoptada para asegurar la implementación del Acuerdo, y lo compara entonces con tres casos reales. Su argumento, por el ejemplo del caso particular a partir del cual se pretende generalizar, cobra fuerza en la medida en que está respaldo por hechos que ya sucedieron, que se pueden comprobar, a pesar de que sean presentados de manera descontextualizada.

Retomando a Perelman y Olbrechts-Tyteca (1958), cuando afirman que la argumentación se basa en premisas que son comunes a quien enuncia y al auditorio, las cuales permiten puntos de acuerdo y sin las cuales no sería posible intercambiar o llegar a consensos, el primer argumento elegido por Uribe Vélez cobra peso

10 A finales del 2014 y principios del 2015, el expresidente y senador Uribe propuso una asamblea constituyente limitada para implementar el Acuerdo de Paz ("Uribe propone", 2015) y relevancia porque parte de una premisa implícita compartida con una parte de la población: con la guerrilla no se puede y no se debe negociar, pues es considerado como un grupo terrorista. La guerrilla se debe rendir ante la ley y ante la institución del Estado. Con lo cual, concluye o reafirma que el proceso de paz es imperfecto. Se observa, por otro lado, cómo las intervenciones de Uribe van aumentando el nivel de agresividad y radicalismo. En cada caso mezcla ideas que habían estado circulando por los medios de comunicación y el recurso a las emociones se reactiva, relacionadas con el miedo por el futuro incierto de las FARC-EP en tanto se habilita su acceso al poder.

En la cartilla, De la Calle se pronuncia sobre esta polémica en el quinto mito y señala:

El Marco Jurídico para la Paz contempla los límites generales de una estrategia integral de justicia transicional que necesariamente tendrá que enmarcarse en las obligaciones internacionales del Estado colombiano. La integralidad se refiere, por un lado, a lograr una aplicación coherente de una serie de distintas medidas judiciales y extrajudiciales para la satisfacción de los derechos de las víctimas a la verdad, la justicia, la reparación y la no repetición; y de otro, a permitir la reintegración sostenible de los ex combatientes a la vida civil.

Todo el esfuerzo que adelanta el Gobierno para lograr la terminación del conflicto debe concluir en un fortalecimiento integral de la justicia y el imperio de la ley en todo el territorio nacional, lo cual es la verdadera garantía de no repetición.

El tratamiento jurídico de quienes hayan participado en graves violaciones a los DDHH en la fase de transición depende de su disposición a reconocer su responsabilidad, hacer frente a sus víctimas y participar activamente en la reconstrucción de la verdad y la reparación de las víctimas.

[...] Más allá del número de procesos penales y los años de privación de la libertad, la verdadera lucha contra la impunidad se concentra en develar qué fue lo que sucedió y por qué; reconocer a las víctimas y repararlas integralmente de manera pronta; y atribuir responsabilidad a los máximos responsables de los crímenes internacionales, a través de mecanismos públicos, de fácil acceso, con decisiones prontas y claras, que le permitan a las víctimas y a la sociedad en general, volver a confiar en las instituciones. (OACP, 2014)

Los argumentos que el Gobierno elige incluir en las cartillas respecto al tema de la impunidad buscan movilizar un sentido común instalado en la población colombiana sobre la eficiencia de los procesos 
penales hegemónicos que reivindican la cárcel como institución central. Esta intención se vincula con uno de los propósitos principales del proceso, asociado a la importancia de la reconstrucción de la verdad de los hechos cometidos durante el conflicto armado, en aras de la satisfacción de los derechos de las víctimas. Si bien en este movimiento de foco (en las víctimas y no únicamente en los "criminales") podría estar de acuerdo una gran parte de la población, incluso quienes simpatizan con los postulados uribistas, la comprensión de la cárcel como el mejor mecanismo para penalizar a quienes violan las leyes sigue siendo el que prima, en contraposición a la posibilidad de "pagar las penas" de manera alternativa, cuando no están relacionadas con la violación al derecho humanitario internacional, con la restricción de la libertad y realizando trabajo comunitario, tal como establece el Acuerdo de Paz.

De esta manera, el Gobierno argumenta a favor del Acuerdo de Paz, y asegura que la guerrilla no saldrá impune luego de la firma del Acuerdo. Para ello se vale de una serie de aclaraciones conceptuales: al inicio del texto citado, comienza clarificando el concepto de "integralidad". Sobre este resalta que contempla varias medidas judiciales y extrajudiciales que están dirigidas a satisfacer los derechos de las víctimas y a garantizar la reintegración de la guerrillerada. Le agrega a esta definición la intención de que, con esta ley, finalmente, se acobije efectivamente a todo el territorio nacional, producto de lo pactado en el Acuerdo Final. Sobre el mecanismo que definieron para pagar las penas por parte de quienes hayan participado en la violación de derechos humanos, vuelven a hacer énfasis en el componente de la verdad a partir del reconocimiento de responsabilidades frente a las víctimas. Con este tipo de argumentos, la OACP intenta desglosar y resumir el contenido del acuerdo parcial sobre la justicia integral para la paz, buscando subrayar el hecho de que el eje está en las víctimas. No resulta una cuestión menor la reafirmación, en este caso, del lenguaje conceptual propio del Acuerdo dado que esta ausencia de traducción a otros lenguajes, en aras de la precisión terminológica, posee consecuencias en los modos en que saberes e identidades son construidos por el dispositivo de pedagogía de paz en su realización por parte del Gobierno.

\section{"Las FARC-EP pagarán penas simbólicas por sus crímenes"}

Como hemos señalado hasta acá, la mayor polémica giró alrededor del "castigo" que debían recibir la las FARC-EP, particularmente los altos mandos, en el marco del proceso de paz. A continuación, presentamos una nueva polémica que giró alrededor de las implicaciones de dicha jurisdicción con respecto a la "rendición de cuentas" ante el Estado y la sociedad colombiana por parte de dicho grupo. En este último proceso de paz con las FARC-EP, se evidenció que uno de los factores que le permitió a este último llegar hasta la firma del Acuerdo Final fue el consenso acerca de buscar soluciones alternativas para que la guerrilla se hiciera cargo de los delitos cometidos y respondiera por ellos, de una manera distinta a la cárcel ordinaria, a la justicia tradicional. No obstante, y como es de esperarse, el Gobierno tampoco estuvo dispuesto desde el principio a que el proceso se redujera a un cese de actividades violentas en el país por parte de esta organización y que ésta saliera "ilesa" legalmente. Para el Gobierno, la guerrilla debía ser juzgada.

Teniendo en cuenta estas salvedades generales, el acuerdo de La Habana en lo relativo a la Justicia Especial para la Paz (JEP), diferencia entre los crímenes de lesa humanidad y otros crímenes, entre quienes fueron responsables directos de estos (como el caso de mandos altos) y quienes los ejecutaron o participaron de manera indirecta. En este orden de ideas, los diversos mecanismos para cumplir la condena por los delitos tienen en cuenta estos diferentes niveles de responsabilidad y de gravedad —en términos de afectación a la comunidad, por ejemplo-, pero también el compromiso con la verdad. Esto quiere decir que quienes cometieron dichos crímenes y acciones violentas deben contar toda su verdad sobre los hechos por los que se les acusa para poder acceder efectivamente a la JEP; es decir, para no pasar por la justicia ordinaria que implica restricción de la libertad en cárceles. Y, por otro lado, establece que la Jurisdicción Especial determine los casos de crímenes de lesa humanidad y las personas que deben ser juzgadas por ellos, quienes, a su vez, serán sometidas a restricción efectiva de la libertad y trabajo para la comunidad.

En la misma lógica de las anteriores polémicas públicas, en cuanto se conoció públicamente este aspecto del Acuerdo, la oposición de derecha salió a la esfera pública a manifestar su desacuerdo. En una de las últimas entrevistas, antes del plebiscito, Uribe dijo lo siguiente sobre la "pena simbólica" con la que se beneficiaría a las FARC-EP:

Esta paz que se está firmando, en la cual, por ejemplo, la FARC, que es campeona mundial de violación de mujeres, que ha reclutado tanta niña, las ha violado, que cuando, las han obligado a entregarle el cuerpo a los cabecillas y han resultado embarazadas, también las han obligado, con fusil, a abortar. Y no van a ir a la cárcel los responsables de esos delitos. No van a ir a la cárcel los responsables de las graves masacres. No van a ir a la cárcel los responsables de los carros bombas, de los burros bombas, de los collares bombas. No van a la cárcel esos cabecillas. Así sea por tiempo corto. Y además les dan derecho de legibilidad política al narcotráfico. Y la FARC es el principal cartel de cocaína del mundo. 
A sus máximos responsables no los extraditan, ni van a la cárcel en Colombia, les dan elegibilidad política. Quiero recordar: nosotros extraditamos cerca de 1200 narcotraficantes durante nuestro gobierno. Entonces, nosotros hemos dicho: esto no va a conducir a una paz estable, a una paz duradera. (NTN24, 2016, 5 de agosto)

La pregunta principal de esta entrevista fue sobre las razones que lo motivaron a él y a la oposición de derecha a impulsar el No en el plebiscito. En este contexto, el hoy senador recurrió nuevamente a los sentimientos y las emociones para desvirtuar el Acuerdo entre el GobjMS y las FARC-EP. Si bien es cierto que a lo largo de los años a esta organización guerrillera se le ha acusado de cometer los crímenes a los que se hace referencia en la entrevista, también lo es que el proceso de paz pretende dilucidar esos hechos. Y en lo que respecta al mecanismo por el cual la guerrilla va a dar cuenta de sus responsabilidades, el expresidente asegura que el hecho de que no vayan a ir a la cárcel o a ser extraditados - como sí se hizo durante su gobierno- significa que no van a ser castigados ante la ley y, por lo tanto, que el castigo va a ser simbólico.

De esta manera, en la primera parte de la transcripción de la entrevista, cuando Uribe se refiere a los hechos cometidos por esta guerrilla, señala: "la FARC, que es campeona mundial de violación de mujeres, que ha reclutado tanta niña, las ha violado, que cuando, las han obligado a entregarle el cuerpo a los cabecillas y han resultado embarazadas". Este fragmento evidencia claramente la estrategia argumentativa escogida por el expresidente en esta intervención, apelando constantemente a las emociones, tratando de generar rechazo, en este caso, frente a la organización guerrillera por el carácter de los hechos. Cierra dicha lista de actos con un argumento que resalta la agresividad de estos: "también las han obligado, con fusil, a abortar", al especificar que la imposición a abortar - lo cual ya resulta agresivose hace con un fusil en mano. Elige, entonces, atacar al Gobierno, por medio de la deslegitimación de la guerrilla como interlocutor de la mesa de conversaciones y no a través de contrargumentos. Otro apartado que pone en evidencia esta estrategia argumentativa es el siguiente: "Y la FARC es el principal cartel de cocaína del mundo", en tanto procura desacreditar a la guerrilla por medio del señalamiento de una de las características impopulares que se le han asignado, refiriéndose a ella como el cartel de cocaína más grande del mundo, a pesar de que se trata de un hecho no confirmado.

Frente a estas versiones, el Alto Comisionado para la Paz, en uno de los videos difundidos el 13 de mayo del 2016, aseguró al respecto que:
Las sanciones serán: actos de reparación, acciones de reparación a las víctimas en los territorios, en condición de restricción efectiva de la libertad hasta por ocho años, para quienes reconozcan toda la verdad de los crímenes cometidos. 0 cárcel, hasta por 20 años, para quienes no lo hagan. Eso no es simbólico, eso es real y es una enorme contribución a la justicia y a la paz. (OACP, 2016)

Esta es una intervención de afirmaciones positivas y de negaciones refutativas ("esto no es simbólico"), en la media en que expone cómo serán las sanciones. A partir de los ejemplos, se pretende explicar por qué no se trata de unas penas simbólicas, sino que, efectivamente, los guerrilleros tendrán que realizar una serie de acciones para resarcir sus "infracciones". Se podría pensar que pretende invertir los contrargumentos del No al plebiscito a partir de datos concretos y, de esta manera, evitar las especulaciones y las generalidades. Para este fin, expone ejemplos de sanciones y resultados esperados concretos como "acciones de reparación a las víctimas en los territorios, en condiciones de restricción efectiva de la libertad hasta por ocho años" o "cárcel, hasta por 20 años, para quienes no [reconozcan toda la verdad de los crímenes cometidos]".

Considerando que esta última polémica pública reseñada aparece de la mano con la anterior, pues se refieren al mismo tópico - la JEP-, el uso pedagógico que se le da a esta última contiene contenido menos conceptual e intenta ir más allá del contenido literal de los acuerdos parciales y del Acuerdo Final, eligiendo un contenido más explícito con respecto a las anteriores respuestas. Esto es, podría ser posible que en este momento del proceso de paz y de las polémicas existiera un grado de impotencia por parte del Gobierno frente a la búsqueda por clarificar los alcances de los acuerdos de la mesa de conversaciones, con lo cual, esta intervención del Alto Comisionado recurre a ejemplos que contienen información más evidente para resaltar el punto que quieren demostrar.

Los ejemplos de las polémicas públicas acá presentados son un reflejo del escenario polémico generado durante todo el proceso de paz y, en particular, de los argumentos que fueron utilizados por los diferentes actores de la oposición, especialmente el uribismo; argumentos que fueron bajando o subiendo de intensidad a largo de los cuatro años que duró la Mesa de Conversaciones y, en particular, en el recorte temporal seleccionado para este análisis. Y, a su, vez, ponen en evidencia el uso pedagógico que se les dio en el marco de la realización del dispositivo pedagógico por parte del Gobierno nacional, negociador de turno. 


\section{La polémica como posibilidad pedagógica}

Podemos analizar el uso pedagógico de estas polémicas a partir tanto de las identidades individuales y colectivas conformadas y (re)construidas, como de los saberes que circularon y se (re)configuraron en relación con su uso pedagógico. Se señalaba al principio de este texto que la polémica se lleva a cabo mediante tres procedimientos: la polarización, la dicotomización y el descrédito. La primera en cuanto fenómeno retórico "puede definirse como un proceso a través del cual un público extremadamente diversificado se fusiona en dos o más grupos fuertemente opuestos y mutuamente excluyentes que comparten gran solidaridad con respecto a valores que el argumentador considera fundamentales" (King y Anderson, citados en Amossy, 2016, p. 28). Esto es, en la medida en que la polémica implica, por lo menos, dos tesis opuestas en confrontación, puede suceder que se concrete en divisiones entre grupos antagonistas en los que cada uno establece su identidad social oponiéndose al otro y convirtiéndolo en símbolo del error y del mal. Esta división entre grupos antagónicos implica un "nosotros" y un "ellos":

[E]l "nosotros" está constituido en los hechos por un "ello"; símbolo de lo que hace posible cualquier "nosotros", ese "ellos" aparece de hecho como una exterioridad constitutiva. [...] [L]as identidades colectivas pueden transformarse siempre en relaciones compatibles, aunque "el antagonismo no puede ser jamás eliminado y él constituye una posibilidad permanente en política". (Mouffe, citada en Amossy, 2017, pp. 35-36)

Es decir que las identidades son constituidas a partir del disenso, del conflicto, de la confrontación, de lo que en un ideal democrático se pretende evitar, evadir u ocultar. Y, en la misma línea, aparece entonces la posibilidad de construcción de identidades políticas, como identidades pedagógicas.

A partir del uso pedagógico que se les dio a las polémicas públicas acá expuestas, se podría decir que la realización del Gobierno procuró formar, por lo menos, dos "nosotros" distintos. Uno de ellos está constituido por el Gobierno colombiano y por las FARC-EP, representado en la Mesa de Conversaciones de La Habana. Esta identidad fue determinada alrededor de la idea de un proceso de negociación para la construcción de un país en paz. A su vez, esta identidad fue reafirmada por el compromiso de las dos partes de reconocer y responder por ciertas responsabilidades de actos cometidos en el marco del conflicto armado. A este "nosotros" se le podría derivar un ("nosotros")' (nosotros prima), el cual va más allá de la mesa de conversaciones como una unidad e incluye, por una parte, esos otros actores sociales y políticos que estuvieron activos durante todo el proceso de paz como movimientos sociales y gremiales y organizaciones de derechos humanos y, por otra parte, a la población colombiana en general, procurando instalar la idea de que en el centro del proceso de negociación estaban las víctimas y la construcción de la paz. Este ("nosotros")' podría denominarse, retomando la nominalización que circuló en los medios de comunicación, como "los amigos de la paz". El "ellos", como exterioridad constitutiva de este primer "nosotros" -incluido el ("nosotros")'一, es encarnado por los discursos que expresaron rechazo al proceso de paz. Este "ellos" fue denominado como "los enemigos de la paz", donde estaban incluidos actores políticos colectivos como el mencionado uribismo.

El segundo "nosotros", que probablemente tuvo menos éxito en su constitución dado el nivel de polarización con el uribismo, es el que el GobjMs intentó constituir o reconfigurar a partir de su realización pedagógica del dispositivo. Esta identidad tiene una historia que va más allá del proceso de paz, a partir de la cual se busca generar vínculos políticos y de afecto con sus antiguos aliados. En la respuesta al primer mito, sobre entregarle el país a la guerrilla, la OACP dice que "no hay que confundir las intervenciones habituales de las FARC a través de las cuales dan a conocer las más disímiles posiciones políticas, con lo que ocurre en la Mesa de Conversaciones" (OACP, 2014). Con esta intervención, como señalamos más arriba, el Gobierno diferencia a esa guerrilla que está sentada en La Habana negociando la salida política del conflicto armado de aquella que ha representado la organización levantada en armas más grande del país, con la que no comparte los presupuestos políticos.

A propósito de la (re)configuración de saberes, podríamos decir que quienes generaron las polémicas públicas, específicamente el uribismo, tuvieron una intención de recuperar y apelar a saberes más relacionados con la experiencia y la emotividad del pueblo colombiano fundado en una gran audiencia nacional. Observamos así una apelación a las emociones como estrategia argumentativa. Estos saberes son recuperados y reconstruidos a partir de la puesta en circulación en las intervenciones en medios de comunicación masiva de un cúmulo de anécdotas y recuerdos sobre los hechos de violencia cometidos por la guerrilla y, así, se busca generar rechazo por parte de toda la sociedad al acuerdo de paz. La reconstrucción de dichos saberes más experienciales pasa por la recuperación positiva de las políticas contrainsurgentes y contraterroristas implementadas durante las administraciones de Uribe, las cuales él considera como benéficas para la sociedad colombiana. 
En lo que respecta a los saberes construidos y transmitidos por la realización del dispositivo de paz del gobierno de Santos, estos estuvieron más relacionados con la divulgación de un saber técnico o científico relativo a los distintos puntos del Acuerdo y a las polémicas públicas en circulación. Este tipo de saber responde a la estrategia del equipo negociador del Gobierno de ceñirse estrictamente a lo que fueron pactando en La Habana, con lo cual, los saberes transmitidos siempre tuvieron una estrecha y evidente relación con los contenidos de los acuerdos parciales. A partir de los documentos escogidos para este análisis de las polémicas, pareciera que este saber científico se encuentra, retomando a Bernstein (2005), descontextualizado ${ }^{11}$ por cuanto no recuperó la cotidianidad ni las tramitaciones locales de las experiencias traumáticas vividas por la población civil durante el conflicto armado en el periodo de tiempo seleccionado, quizás con excepción de la última polémica donde se vislumbra la incorporación de un contenido más explícito referido a las sanciones que se le aplicarán a la guerrilla.

Es decir, estos materiales no ponen en evidencia ningún diálogo directo y real con la población colombiana, donde sus contenidos entraran en un proceso de recuperación de saberes más experienciales y vivenciales, cuestión que podría esperarse en tanto los puntos de la agenda de negociación parten de las problemáticas que se consideran que han permitido el conflicto armado entre el Estado y la guerrilla de las FARC-EP. A este respecto, no hay evidencia de estrategias pedagógicas locales, en el marco de esta realización - y tampoco de la de la guerrilla - a partir de las cuales hubiera una adaptación de los acuerdos parciales a las realidades locales. Es posible que esta carencia de concreción de los materiales pedagógicos responda a un criterio de inmediatez en la necesidad de socialización de los acuerdos de cara a los tiempos impuestos por el plebiscito y, en este sentido, a que el Gobierno tuviera la obligación constitucional de hacer llegar esta información a todo el territorio nacional —más allá de si lo logró efectivamente o no--, lo cual implicaba un destinatario amplio y, en consecuencia, heterogéneo.

Retomando los postulados de la retórica y la teoría de la argumentación, señalados por Amossy (2016), a partir de los cuales se rechaza la polémica por obstruir el objetivo utópico democrático - el consenso-, a modo de recopilación, podríamos señalar que los argumentos, enmarcados en las polémicas retomadas y utilizadas por la realización del dispositivo pedagógico del Gobierno, tuvieron el siguiente trayecto:

11 El autor entiende por saberes descontextualizados aquellas situaciones pedagógicas que carecen de procedimientos de apropiación y reformulación de un discurso, en tanto el objetivo deja de ser transmitirlo en contextos distintos al de su contexto de producción. primero constituyeron polémicas -instaladas por parte de la oposición al proceso de paz-, luego una estrategia pedagógica -el uso pedagógico de dichas polémicas públicas en el marco de la realización del dispositivo por parte del Gobierno-y, después, se convirtieron en acuerdos - materializados en el Acuerdo Final y Definitivo-. Es decir, construyeron una nueva versión del acuerdo de paz. En esta medida, se podría decir que en el caso del dispositivo de la pedagogía de paz se manifestó una excepcionalidad con respecto a la búsqueda de consenso, puesto que durante el proceso de paz se generaron varias situaciones discursivas polémicas, no obstante, resultaron en acuerdos entre el Gobierno y la guerrilla de las FARC-EP. De alguna manera, sería posible señalar que con los diálogos de paz se estaba buscando una democracia sin conflicto armado y con ella su horizonte utópico: el acuerdo, no solo entre el Gobierno y las FARC-EP, también con la sociedad civil, el pueblo colombiano, como se insistió a lo largo del proceso. Por esto, fue fundamental que parte de las polémicas se "convirtieran" en acuerdos. A este respecto, cabe mencionar el segundo y definitivo acuerdo de paz, el cual incluyó ciertas propuestas - argumentos-de la campaña por el No. Desde la misma mesa de conversaciones (Gobierno y guerrilla), al final, incluyeron varios de los puntos que atacaban el Acuerdo Final, los cuales fueron reconocidos como argumentos válidos y no únicamente como mentiras infundadas.

Por último, si bien consideramos que la polémica misma podría tener un componente pedagógico no intencional, pues a través de esta se transmiten valores y saberes a la sociedad, acá analizamos el uso deliberado que se le dio a ciertas polémicas públicas desde el dispositivo de paz. De este modo, uno de los aspectos que resultan relevantes de señalar, a partir de esta reconstrucción y análisis del uso pedagógico de las polémicas públicas reseñadas, es el carácter instrumental, con fines pedagógicos, de las polémicas públicas por parte del Gobierno. Dicha inclusión de polémicas fue introducida en su realización a través de los materiales de difusión y a partir de una dimensión técnica traducida en la prevalencia de saberes científicos descontextualizados. De este modo, desde la realización del Gobierno buscaron construir, en primera medida, un "nosotros" —incluido el ("nosotros")' - que fortaleciera el proceso de paz y, de alguna manera, humanizara a la guerrilla -interlocutor-, corriéndola del imaginario de "terroristas" con quienes no se podía dialogar ni negociar. Y, en segunda medida, no dejaron de lado ese antiguo "nosotros" que se fue deteriorando y desvaneciendo al pasar de los años y que, en el marco de las rondas de conversación, no era favorable para el fin último de estas: la refrendación y posterior implementación exitosa del Acuerdo Final para la Paz. 


\section{Referencias}

Amossy, R. (2016). Por una política del dissensus: las funciones de la polémica. En A. S. Montero (comp.), El análisis del discurso polémico. Disputas, querellas y controversias (pp. 25-38). Prometeo Libros.

Amossy, R. (2017). Apología de la polémica. Prometeo Libros.

Amstutz, J., Mazzarantani, E. y Paillet, M. (2005). Pedagogía de la paz. Construir la convivencia manejando adecuadamente los conflictos. Edualter. http://www.edualter. org/material/pedagogia_de_la_paz.pdf.

Bernstein, B. (2005). Clases, códigos y control II. Hacia una teoría de las transmisiones educativas. Akal. (Original publicado en 1975).

Gobierno de Colombia y FARC-EP. (2016, 12 de mayo). Comunicado Conjunto \#69. OACP. http://www.altocomisionadoparalapaz.gov.co/procesos-y-conversaciones/documentos-y-comunicados-conjuntos / Documentos\%20compartidos/Comunicado-Conjunto-69-12-de-mayo-de-2016.pdf

Gobierno nacional y FARC-EP (2016, 26 de agosto). Acuerdo Final para la terminación del conflicto y la construcción de una paz estable y duradera. Mesa de Conversaciones en La Habana, Cuba. http://www.altocomisionadoparalapaz.gov.co/procesos-y-conversaciones/Paginas/ Texto-completo-del-Acuerdo-Final-para-la-Terminacion-del-conflicto.aspx

Grinberg, S. (2008). Educación y poder en el siglo XXI. Gubernamentalidad y pedagogía en las sociedades de gerenciamiento. Miño y Dávila.

Grinberg, S. (2011, agosto). Gubernamentalidad y educación en tiempos de gerenciamiento. Reflexiones en torno de la experiencia de los dispositivos pedagógicos en contextos de extrema pobreza urbana. VIII Encuentro de Cátedras de Pedagogía de Universidades Nacionales Argentinas, La Plata, Argentina.

Kerbat-Orecchioni, C. (2016). Sarkosy polemista: la "descalificación cortés" del adversario. En A. S. Montero (comp.), en El análisis del discurso polémico. Disputas, querellas y controversias (pp. 97-121). Prometeo Libros.

Langer, E. (2013). Los dispositivos pedagógicos en las sociedades de control: prácticas de resistencia de estudiantes y regulación de las conductas en escuelas secundarias en contextos de pobreza urbana del Partido de San Martín [tesis de doctorado no publicada, Universidad de Buenos Aires].
Le van a entregar el país a las Farc por meterme a la cárcel: Uribe al fiscal. (2015, 28 de septiembre). El Espectador. https://www.elespectador.com/noticias/politica/ le-van-entregar-el-pais-farc-meterme-carcel-uribe-alfi-articulo-589358

Montero, A. S. (2016). El discurso polémico. Disputas, querellas y controversias. Prometeo Libros.

NTN24. (2016, 13 DE MAYO). Uribe y Zuluaga rechazan blindaje jurídico de acuerdos de paz entre Colombia y las FARC [video]. YouTube. https://www.youtube.com/ watch?v=Rm0Jm3PSQpw

NTN24. (2016, 5 de agosto). Campañas por el Sí y el No en plebiscito, el nuevo pulso político en Colombia. I [video]. YouTube. https://www.youtube.com/ watch?v=adJT04LMVNI

Oficina del Alto Comisionado para la Paz. (2014). Entérese del Proceso de Paz.

Oficina del Alto Comisionado para la Paz. (2016). Mitos urbanos sobre el proceso de paz [videos]. YouTube. https: //www.youtube.com/watch?v=0nEqv3x4ERA\&list=PLO_sOiuOzMAEHQWFdilyyLo2VBrnlX7Cx

Palumbo, M. M. (2014). Las prácticas político-pedagógicas de los movimientos populares urbanos. El caso del Movimiento Popular La Dignidad en la Ciudad Autónoma de Buenos Aires (2012-2013) [tesis de maestría no publicada, Universidad de Buenos Aires].

Palumbo, M. M. (2017). Dinámicas de construcción de subjetividades políticas y epistémicas en dispositivos pedagógicos de formación política. Un estudio en movimientos populares multisectoriales del Área Metropolitana de Buenos Aires (2011-2015). http:// revistascientificas.filo.uba.ar/index.php/tesis/article/ view/5365

Perelman, C. y Olbrechts-Tyteca, L. (1958). La nouvelle rhétorique; Traité de l'Argumentation. Presses Universitaires de France.

Simons, M. y Masschelein, J. (2017). Subjetivación gubernamental, política y pedagogía. Foucault con Rancière. En M. Simons, J. Masschelein y J. Larrosa (eds.), Jacques Rancière. La educación pública y la domesticación de la democracia (pp. 107-145). Miño y Dávila.

Uribe propone convocar a una Constituyente limitada a acuerdos de paz. (2015, 27 de octubre). El Tiempo. https://www.eltiempo.com/archivo/documento/ CMS-16414838

Uribe: "Zuluaga es lo contrario al castro-chavismo de Maduro, Santos, Petro y las Farc". (2014, 15 de mayo). Iberoamérica Central de Noticias. https://www.icndiario.com/2014/05/uribe-zuluaga-es-lo-contrario-alcastro-chavismo-de-maduro-santos-petro-y-las-farc/ 